\title{
Value of 3T Susceptibility-Weighted Imaging in the Diagnosis of Multiple Sclerosis
}

\author{
(D) M.A. Clarke, (DD. Pareto, (D). Pessini-Ferreira, (D) G. Arrambide, (D) M. Alberich, (D)F. Crescenzo, (D). Cappelle, (D) M. Tintoré, \\ (D). Sastre-Garriga, (DC. Auger, (D) X. Montalban, (D) N. Evangelou, and (D) À. Rovira
}

\begin{abstract}
BACKGROUND AND PURPOSE: Previous studies have suggested that the central vein sign and iron rims are specific features of MS lesions. Using 3T SWI, we aimed to compare the frequency of lesions with central veins and iron rims in patients with clinically isolated syndrome and MS-mimicking disorders and test their diagnostic value in predicting conversion from clinically isolated syndrome to MS.
\end{abstract}

MATERIALS AND METHODS: For each patient, we calculated the number of brain lesions with central veins and iron rims. We then identified a simple rule involving an absolute number of lesions with central veins and iron rims to predict conversion from clinically isolated syndrome to MS. Additionally, we tested the diagnostic performance of central veins and iron rims when combined with evidence of dissemination in space.

RESULTS: We included 112 patients with clinically isolated syndrome and 35 patients with MS-mimicking conditions. At follow-up, 94 patients with clinically isolated syndrome developed MS according to the 2017 McDonald criteria. Patients with clinically isolated syndrome had a median of 2 central veins (range, 0-19), while the non-MS group had a median of 1 central vein (range, 0-6). Fiftysix percent of patients who developed MS had $\geq 1$ iron rim, and none of the patients without MS had iron rims. The sensitivity and specificity of finding $\geq 3$ central veins and/or $\geq 1$ iron rim were $70 \%$ and $86 \%$, respectively. In combination with evidence of dissemination in space, the 2 imaging markers had higher specificity than dissemination in space and positive findings of oligoclonal bands currently used to support the diagnosis of MS.

CONCLUSIONS: A single 3T SWI scan offers valuable diagnostic information, which has the potential to prevent MS misdiagnosis.

ABBREVIATIONS: CDMS = clinically definite MS; CIS = clinically isolated syndrome; $C V$ = central vein; DIS = dissemination in space; DIT = dissemination in time; $\mathbb{I R}=$ iron rim; $N P V=$ negative predictive value; $O C B=$ oligoclonal band; $P P V=$ positive predictive value

$\mathrm{S}$ diagnosis is based on typical clinical symptoms and radiologic findings, and it incorporates the principles of

Received December 19, 2019; accepted after revision March 19, 2020

From the Vall d'Hebron Research Institute (M.A.C., D.P., M.A., M.T.,J.S.-G., C.A., X.M A.R.), Barcelona, Spain; Section of Neuroradiology, Department of Radiology (D.P. L.P.-F., C.A., A.R.), Hospital Universitari Vall d'Hebron, Universitat Autònoma de Barcelona, Barcelona, Spain; Department of Neurology-Neuroimmunology (G.A., M.T., J.S.-G., X.M.), Centre d'Esclerosi Múltiple de Catalunya (Cemcat), Hospital Universitari Vall d'Hebron, Universitat Autònoma de Barcelona, Barcelona, Spain; Department of Neurosciences, Biomedicine and Movement Sciences (F.C.), University of Verona, Verona, Italy; Division of Radiology (S.C.), University Hospital Leuven, Leuven, Belgium; Division of Neurology (X.M.), St. Michael's Hospital, University of Toronto, Toronto, Ontario, Canada; and Division of Clinical Neuroscience (N.E.), University of Nottingham, Nottingham, UK.

The European Center for Treatment and Research in Multiple Sclerosis and Magnetic Resonance Imaging in Multiple Sclerosis provided funding for M.A.C.'s fellowship. Furthermore, we acknowledge the "Red Española de Esclerosis Múltiple" (RD07/0060; RD12/0032) and the project PI18/00823, which are sponsored by the Fondo de Investigación Sanitaria, the Instituto de Salud Carlos III, the Ministry of Economy and Competitiveness in Spain, and the "Ajuts per donar Suport als Grups de Recerca de Catalunya (2009 SGR 0793)," which is sponsored by the "Agència de Gestió d'Ajuts Universitaris i de Recerca" of the Generalitat de Catalunya in Spain demonstration of demyelinating lesions disseminated in space (DIS) and time (DIT). Radiologically, DIS is demonstrated by the presence of $\geq 1$ T2-hyperintense lesion characteristic of MS in $\geq 2$ of the following CNS topographies: periventricular, cortical, or juxtacortical; infratentorial; and spinal cord; and DIT is demonstrated by the simultaneous presence of gadolinium-enhancing and nonenhancing lesions on a single scan or by a new T2 lesion compared with a previous MR imaging scan. Following the 2017 revisions to the McDonald criteria, a positive finding on lumbar

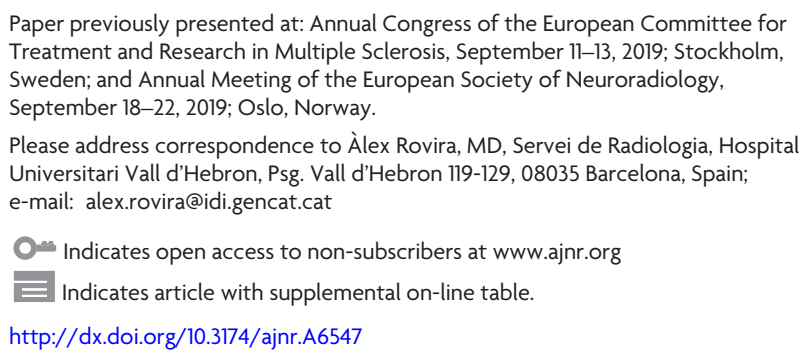


puncture can be used to diagnose MS in the absence of DIT. ${ }^{1}$ This has led to an increase in sensitivity but a decrease in specificity, compared with the 2010 criteria., ${ }^{2,3}$ As a result, MS-specific imaging markers are needed to improve the diagnostic process and prevent overdiagnosis.

SWI is an MR imaging technique sensitive to paramagnetic compounds that distort the local magnetic field, such as deoxyhemoglobin and iron. ${ }^{4,5}$ Recently, the role of SWI in MS has gained attention because it offers additional information about MS WM lesions, which cannot be appreciated on conventional T1- and T2-weighted images currently used to diagnose and monitor patients. ${ }^{6,7}$

Evidence from $7 \mathrm{~T}$ studies and recently from $3 \mathrm{~T}$ and $1.5 \mathrm{~T}$ studies $^{8-13}$ shows that MS lesions form around small veins, a phenomenon termed "the central vein (CV) sign." Studies of patients with established disease have proposed a $40 \%$ threshold of WM lesions with CVs to differentiate MS and other disorders that can mimic MS on MR imaging. ${ }^{14-16}$ Similarly, lesions with hypointense rims, likely reflecting iron deposition within the microglia and macrophagic cells at the edge of some chronic MS lesions, the so-called iron rims (IRs), have been identified on SWI in all subtypes of MS. ${ }^{7,17}$ However, this imaging feature seems to be absent in other diseases such as neuromyelitis optica spectrum disorder, ${ }^{18}$ Susac syndrome, ${ }^{19}$ and ischemic lesions. ${ }^{20}$ This finding suggests that both CVs and IRs might be specific features of MS lesions, which could be applied diagnostically.

However, most studies have assessed the CV sign and the IRs separately and, so far, have mainly been performed on small populations of patients with already-established diagnoses, using 7T scanners and/or sequences not commonly used in clinical practice. In this study, we aimed to assess the frequency of CVs and IRs detected on unenhanced SWI acquired on a clinical 3T scanner in patients with typical clinically isolated syndrome (CIS) and MS-mimicking disorders. Additionally, we aimed to test their usefulness as diagnostic imaging markers of MS lesions in patients at the earliest stages of the disease. We hypothesized that patients who went on to develop MS would have a higher number of lesions with CVs and IRs compared with patients who did not.

\section{MATERIALS AND METHODS Ethics}

This study received approval from the Clinical Research Ethics Committee at the Vall d'Hebron University Hospital (PR(AG) 302/2018). All patients signed written informed consent.

\section{Patients}

We recruited patients between October 2010 and February 2019. All scans were acquired as part of routine, clinical assessment.

Two groups of patients were recruited using consecutive sampling. The first one is part of an ongoing cohort study described previously $^{21,22}$ and included a prospective cohort of patients younger than 50 years of age with a typical CIS suggestive of CNS demyelination, scanned within 3-5 months of the first clinical attack (CIS group). Sixteen patients with CIS included in this study have been previously reported in a cross-sectional study of the CV sign. ${ }^{23}$ The second group comprised patients with WM multifocal abnormalities not attributed to MS scanned either before or after being formally given a non-MS diagnosis (non-MS group). We excluded any patients without SWI or T2-weighted FLAIR images, with images acquired using a different set of SWI and/or FLAIR protocols, and with scans of insufficient quality for analysis. Neurologists and/or radiologists independently provided the clinical diagnoses for the patients included in this study and were blinded to any study results reported here.

\section{Immunoglobulin G Oligoclonal Bands}

Intrathecal immunoglobulin oligoclonal band (OCB) testing was performed in patients with CIS within 3 months of the first clinical attack and in some of the non-MS group (if requested by the patient's neurologist as part of the clinical work-up). OCBs were determined using agarose isoelectric focusing combined with immunoblotting in the CSF and serum. ${ }^{24}$

\section{MR Imaging Acquisition}

All MR images were acquired on a 3T Magnetom Trio MR imaging system (Siemens) with a 12-channel phased array head coil and a whole-body transmit coil. The following sequences were obtained in all the patients: 3D axial gradient-echo SWI without contrast $\left(\mathrm{TR}=33 \mathrm{~ms}, \mathrm{TE} 1=6.08 \mathrm{~ms}, \mathrm{TE} 2=24.6 \mathrm{~ms}\right.$, flip angle $=15^{\circ}$, matrix size $=288 \times 384 \times 104$, voxel size $=0.65 \times 0.65 \times 3.0 \mathrm{~mm})$; and transverse 2D-T2-FLAIR $(\mathrm{TR}=9000 \mathrm{~ms}, \mathrm{TE}=87 \mathrm{~ms}$, $\mathrm{TI}=2500 \mathrm{~ms}$, flip angle $=119^{\circ}$, matrix size $=412 \times 512 \times 46$, voxel size $=0.49 \times 0.49 \times 3.0 \mathrm{~mm})$ or sagittal 3D-FLAIR $(\mathrm{TR}=$ $5000 \mathrm{~ms}, \mathrm{TE}=394 \mathrm{~ms}, \mathrm{TI}=1800 \mathrm{~ms}$, flip angle $=120^{\circ}$, matrix size $=240 \times 256 \times 176$, voxel size $=1.0 \times 1.0 \times 1.0 \mathrm{~mm}) .3 \mathrm{D}$ FLAIR sequences were reconstructed in the axial plane using 3mm-thickness contiguous slices.

\section{Image Processing and Analysis}

Each patient's FLAIR and susceptibility-weighted images were coregistered using SPM12 software (http://www.fil.ion.ucl.ac.uk/spm/ software/spm12), and 3D Slicer, Version $4.10 .0^{25}$ (http://www.slicer. org), was used to assess the images. Brain lesions were identified on the T2-FLAIR images and were analyzed for the presence of both CVs and IRs on the axial plane of the SWI.

For the analysis of CVs, we followed the guidelines described by the North American Imaging in Multiple Sclerosis Cooperative; ${ }^{11}$ however, we included confluent lesions in our analysis to assess both CVs and IRs in all the analyzable lesions and to calculate their combined frequency. If a confluent lesion had a single CV or its "fingers" had CVs, we classed that lesion as positive for a CV. IRs were identified as areas of hypointense ringlike signal, which corresponded to the edge of the lesion, encircling it fully or partially. We also recorded information about lesions with scattered iron deposition in the form of hypointense iron "dots" on SWI, described previously. ${ }^{26} \mathrm{We}$ excluded lesions that were $<3 \mathrm{~mm}$ in their shortest axis, lesions located infratentorially, and lesions that were not fully visible on the SWI. For each lesion, we recorded the topography: juxtacortical, periventricular, subcortical, or deep gray matter.

Image analysis was performed by M.A.C. (with 5 years' experience in MS lesion analysis). A subset of 25 randomly selected scans was analyzed by a second rater (F.C.) to calculate interrater 
agreement and determine the effect of rater experience on identifying the lesion features of interest. The second rater (a neurology resident with 4 years' experience) had no previous experience in identifying CVs and IRs and underwent training under the supervision of M.A.C. before analysis. Both raters were blinded to all clinical information at the time of the analysis.

\section{Study Design}

Our study included 3 main analyses. Data from both patients with CIS and non-MS were used in the first part of the analysis, and we subsequently focused on the CIS population only (Parts 2 and 3).

Part 1: Frequency of CVs and IRs in Patients with CIS and NonMS. First, we compared the number of lesions with IRs, iron dots, and CVs between the CIS and non-MS groups to quantify the frequency of these lesion features in the 2 populations.

Part 2: Diagnostic Value of CVs and IRs. We used information about the frequency of IRs, iron dots, and CVs in the CIS group

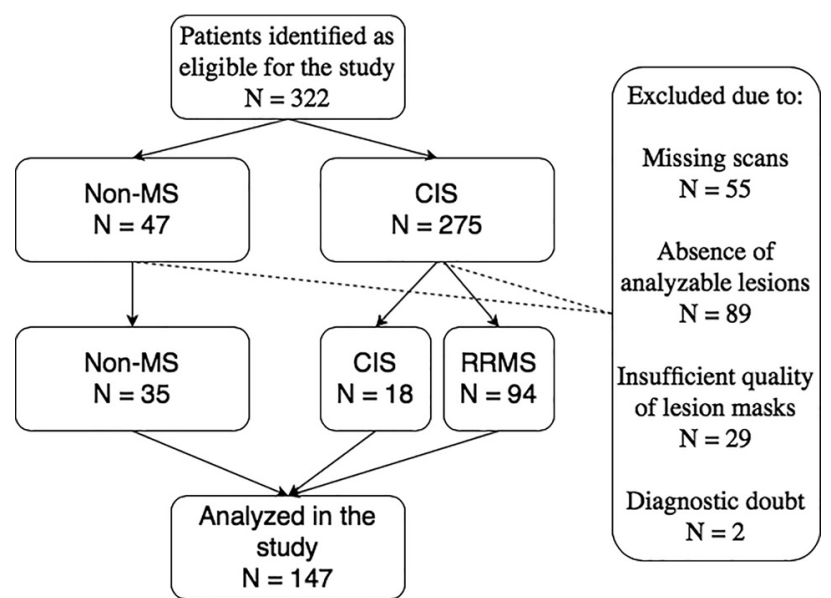

FIG 1. Patient flow diagram showing how patients were selected for the study and the reasons for exclusion. We excluded patients for the following reasons: 1) missing either their SWI or T2-FLAIR scan; 2) lesions $<3 \mathrm{~mm}$ or infratentorial lesions that could not be identified on the SWI due to artifacts; 3) automated lesion masks not passing quality checks; and 4) diagnostic doubt by the end of the study follow-up. RRMS indicates relapsing-remitting multiple sclerosis.

\section{Demographic and clinical data of the patients included in the study}

\begin{tabular}{|c|c|c|}
\hline & CIS Group $(n=112)$ & Non-MS Group $(n=35)$ \\
\hline Mean age (SD, range) (yr) & $35.4(7.9,19-49)$ & $41.7(11.5,20-67)$ \\
\hline Sex, female No. (\%) & 70 (70.5\%) & $23(65.7 \%)$ \\
\hline $\begin{array}{l}\text { Clinical diagnosis at the end of } \\
\text { the study No. (\%) }\end{array}$ & $\begin{array}{l}\mathrm{MS}=94(83.9 \%) \\
\quad \text { (including CDMS }=42 \\
\quad[37.5 \%]) \\
\mathrm{CIS}=18(16.1 \%)\end{array}$ & $\begin{array}{l}\text { Autoimmune disease }=13(37.1 \%) \\
\text { Vascular disease }=8(22.9 \%) \\
\text { Incidental findings }=1(2.9 \%) \\
\text { Infectious disease }=1(2.9 \%) \\
\text { Headache }=3(8.6 \%) \\
\text { Other }=9(25.7 \%)\end{array}$ \\
\hline $\begin{array}{l}+/- \text { OCBs No. (not } \\
\text { performed) }\end{array}$ & $80 / 21$ (11) & $2 / 15(18)$ \\
\hline Median EDSS (range) & $1.5(0-4.5)$ & NA \\
\hline Median WM lesion No. (range) & $4(1-31)$ & $7(1-31)$ \\
\hline
\end{tabular}

Note:-EDSS indicates Expanded Disability Status Scale; NA, not available. to perform an exploratory analysis of the diagnostic value of using an absolute number of lesions with IRs and CVs to predict conversion to MS. We included only patients with a minimum of 3 years of follow-up or a confirmed diagnosis of MS according to the 2017 McDonald criteria.

Part 3: Dissemination in Space + Analysis. We assessed the diagnostic value of IRs, iron dots, and CVs in combination with evidence of dissemination in space (DIS) by testing the following proposed criteria:

- DIS + IR: evidence of DIS and simultaneous presence of rim + and rim - lesions

- DIS + iron: evidence of DIS, and rim + and rim - lesions or iron dots

- DIS + CVs: evidence of DIS and lesions with central veins

We compared the results with the performance of baseline DIS and positive OCBs (DIS + OCB), the simultaneous presence of gadolinium-enhancing and nonenhancing lesions (DIS + gadolinium Gadobutrol [Gadovist, Bayer]), and DIS + any of the above (IR, iron, $2 \mathrm{CVs}$, OCBs, and/or gadolinium). We assessed the diagnostic value of the DIS + criteria compared with the following outcomes: radiologic conversion to MS (at baseline or during followup) and the Poser criteria (clinically definite MS [CDMS] with evidence of 2 clinical attacks separated in time and in space). We included only patients with a minimum of 3 years of follow-up or with a positive outcome (DIS + DIT or Poser criteria).

\section{Statistical Analysis}

Statistical analysis was performed using SPSS, Version 24, and the diagnostic performance was assessed using MedCalc for Windows, Version 15.0 (MedCalc Software). $P<.05$ was used to indicate statistically significant results.

Interrater agreement was calculated separately for lesions with CVs and IRs using the intraclass correlation coefficient (2-way mix model, single measures, absolute agreement) in a small sample of randomly chosen scans.

We selected the minimum number of lesions with CVs and IRs for a diagnosis to ensure high specificity. The sensitivity, specificity, accuracy, positive predictive value (PPV), and negative predictive value (NPV) with 95\% confidence intervals were calculated for each proposed index test. We also tested for the location preference of lesions with CVs and IRs using a chi-square test. A Cox regression was used to calculate the risk of conversion to MS for each of the proposed DIS + criteria. We used patients with CIS not fulfilling the given criteria as the reference group.

\section{RESULTS}

\section{Patients}

One hundred twelve patients with CIS and 35 in the non-MS group were included in the study. A patient flow diagram can be seen in Fig 1. Demographic and clinical data are shown in the Table. In the CIS group, the mean follow-up 


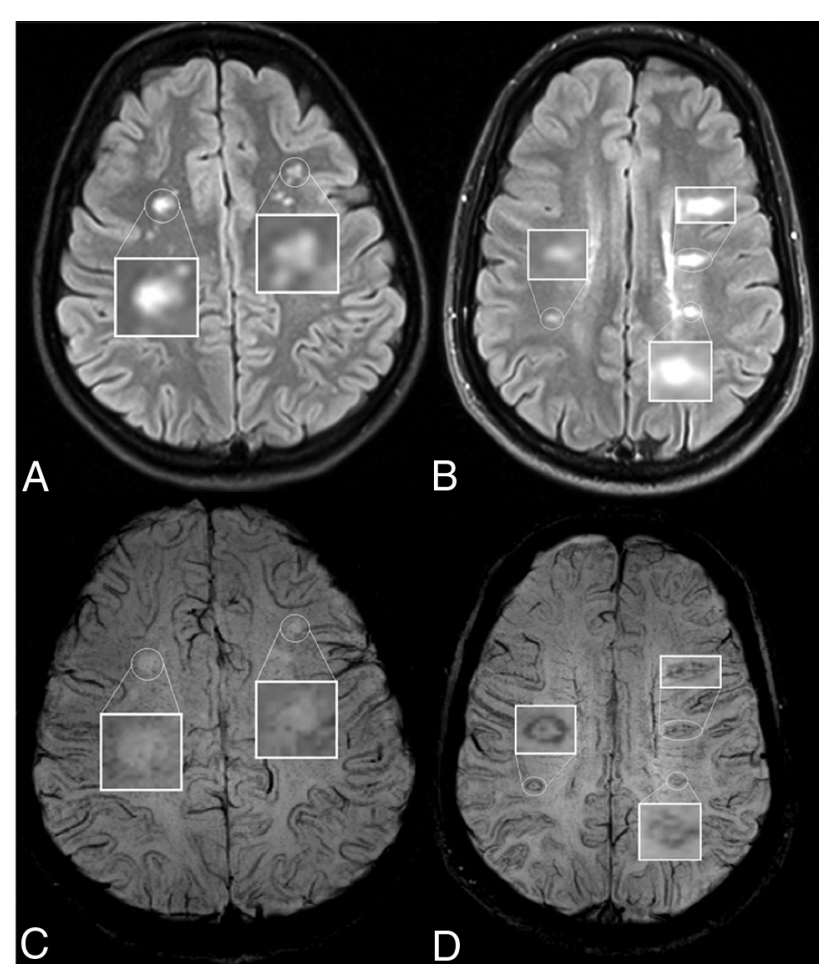

FIG 2. Lesion appearance on axial T2-FLAIR (upper row) and the corresponding susceptibility-weighted (lower row) images in patients with Sjögren disease $(A$ and $C)$ and $M S(B$ and $D)$. The patient with Sjögren disease has no visible CVs or IRs on the SWI. The patient with MS has clearly visible IRs, which correspond to the lesion edges visible on the T2-FLAIR. CVs are also visible inside the lesions as hypointense dots or lines.

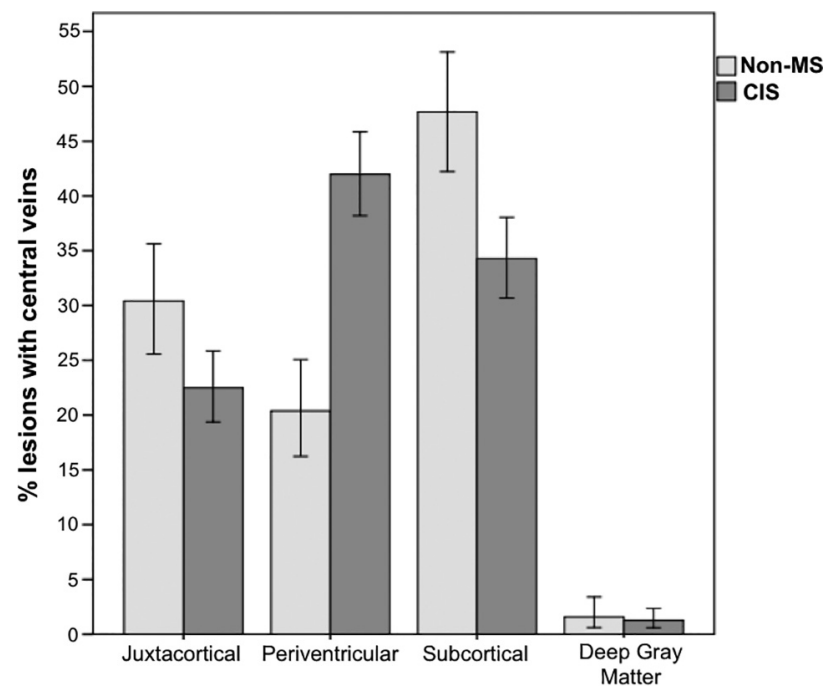

FIG 3. The location of lesions with central veins in the CIS and nonMS groups. Error bars represent standard error.

time was $4.6 \pm 2$ years. During that period, 94 patients (84\%) received a diagnosis of MS according to the $2017 \mathrm{McD}$ onald criteria, including 42 (37.5\%) who converted to CDMS (Poser criteria). ${ }^{27}$ During the follow-up, 24 patients with CIS did not undergo diseasemodifying therapy and 84 did, with data missing for 4 patients. In the non-MS group, 19 individuals (54\%) did not have an established diagnosis at the time of the scan, and the mean follow-up of that group of patients was $4.7 \pm 2$ years. For the non-MS group with an already-established diagnosis before the scan $(n=16)$, the median disease duration was 2 years (range, $0-49$ years).

The non-MS diagnoses included the following groups of diseases: autoimmune (anti-myelin oligodendrocyte glycoproteinassociated disease, Susac syndrome, anti-aquaporin-4 + neuromyelitis optica spectrum disorder, primary antiphospoholipid syndrome, CNS vasculitis, Sjögren, Sjogren's syndrome, neurosarcoidosis), small vessel vascular disease, infectious disease (human T-cell leukemia virus, type 1-associated myelopathy), headache (including migraine), nonspecific leukoencephalopathy, and incidental white matter findings in healthy subjects or unrelated to clinical symptoms (nonspecific paresthesias or visual symptoms, cranial nerve palsy, or neuralgia). A diagnosis of MS had been explicitly excluded by their neurologists in all these cases.

\section{Lesion Analysis}

In total, we analyzed 955 focal WM lesions; 636 in the CIS group and 319 in the non-MS group. Figure 2 shows sample lesions with CVs and IRs and without them.

Regarding the interrater agreement, the intraclass correlation coefficient based on the number of lesions with CVs was 0.84 (95\% CI, 0.67-0.93), and for the number of lesions with IRs, it was 0.84 (95\% CI, 0.64-0.93).

Part 1: Frequency of CVs and IRs in Patients with CIS and NonMS. In the CIS group, 410 lesions (64.5\%) had a CV, while only 53 (16.6\%) had a CV in the non-MS group. Fifty-six (50\%) patients with CIS had $\geq 3$ lesions with CV versus seven (20\%) in the nonMS group. Figure 3 shows the location of lesions with CVs in the CIS and non-MS groups. The difference in the distribution of lesions with CVs in the CIS group, assessed by a chi-square test, was significant $\left(\mathrm{X}^{2}(3)=169.805, P<.001\right)$, showing preference for periventricular and subcortical locations. Figure 4 shows the differences in the proportions and number of lesions with CVs between the 2 groups. None of the non-MS group reached the previously proposed $40 \%$ threshold of WM lesions with CVs (range, 0\%-37.5\%).

One hundred twenty-seven lesions (19.9\%) in the CIS group had an IR, while none of the lesions in the non-MS group had one. Of all patients with CIS, $47.3 \%$ had at least 1 lesion with an IR and none of the patients who remained with CIS at the end of the study both according to the $2017 \mathrm{McD}$ onald criteria and the Poser criteria had any lesions with IRs. Of patients without IRs, $63.6 \%$ subsequently initiated disease-modifying therapies compared with $94.3 \%$ of patients with IRs.

More than half of all the lesions with IRs were located periventricularly, demonstrating a significant location preference $\left(\chi^{2}(3)=\right.$ 86.4, $P<.001$ ), and three-quarters of all the patients with IRs had at least 1 periventricular lesion with an IR; $13.2 \%$ of all CIS lesions had both a CV and an IR. See Fig 5 for a summary of the incidence and location of lesions with IRs in our study.

Forty-six CIS lesions (7.2\%) and 16 non-MS lesions (5\%) had an iron dot. For both the CIS and non-MS groups, the median number of lesions with iron dots was 0 (range, 0-3). Twenty-four patients with CIS and 8 with non-MS had at least 1 lesion with an iron dot. 

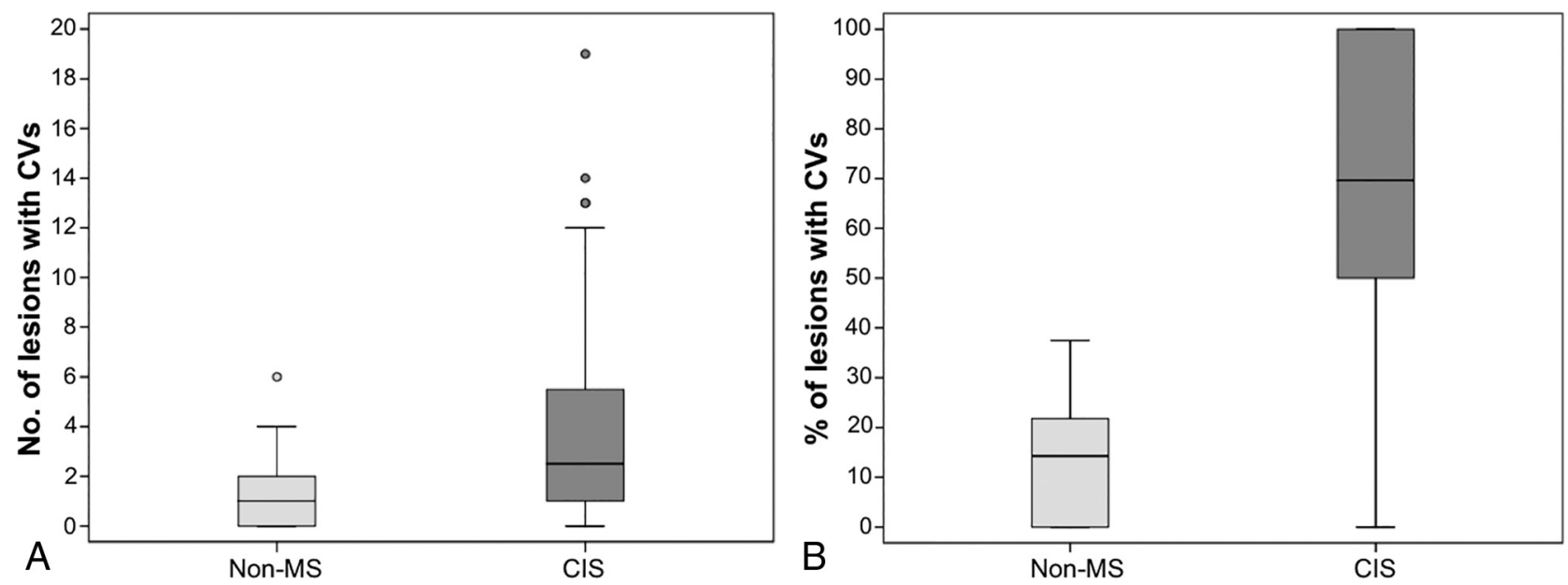

FIG 4. Summary of the incidence of lesions with CVs in the CIS and non-MS groups. A, The number of lesions with CVs (per patient) in the 2 groups. $B$, The percentage of lesions with CVs (per patient) in the 2 groups.
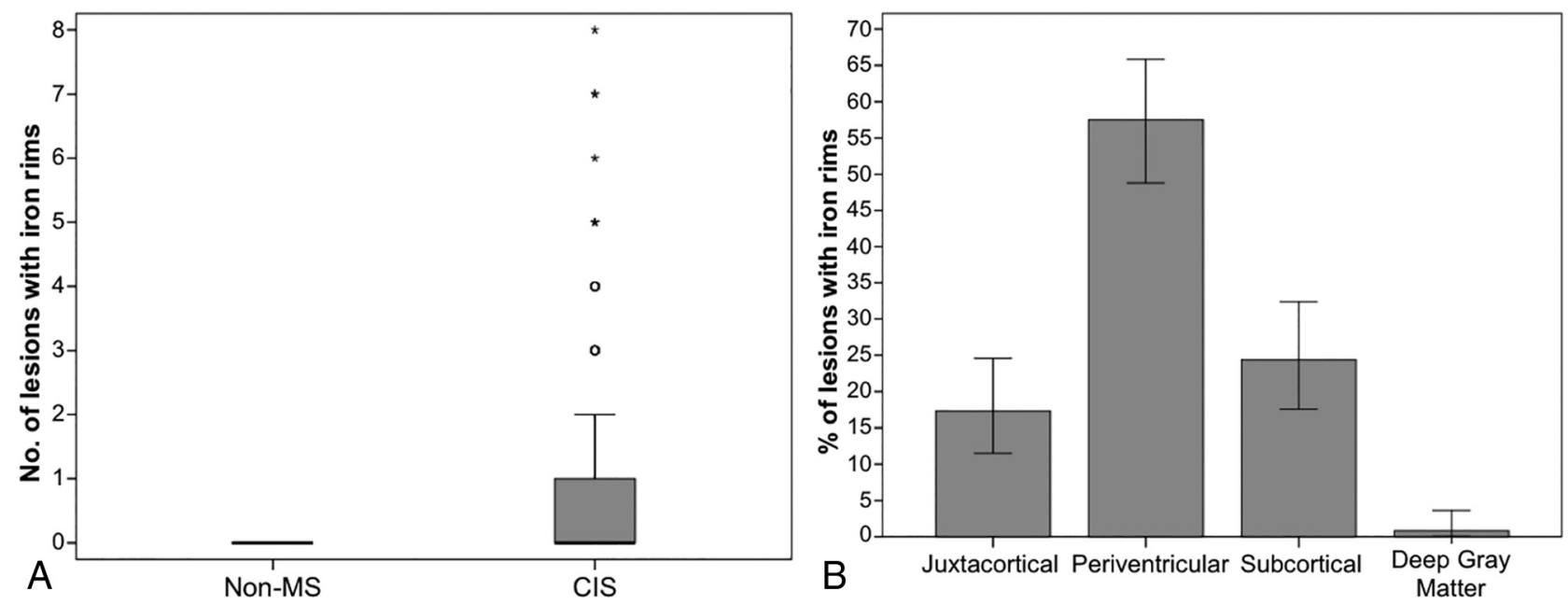

FIG 5. Summary of the incidence of lesions with iron rings in the CIS and non-MS groups. $A$, The number of lesions with iron rings (per patient) in the 2 groups. $B$, The location of lesions with iron rings in the CIS group.

For analyses presented in Parts 2 and 3, we excluded 4 patients with CIS who did not have a minimum of 3 years of follow-up or a confirmed diagnosis of MS according to the 2017 McDonald criteria.

Part 2: Diagnostic Value of CVs and IRs. The presence of 3 lesions with $\mathrm{CVs}$ and/or 1 lesion with an IR on the baseline SWI scan resulted in $70.2 \%$ sensitivity (95\% CI, 59.9\%-79.2\%) and $85.7 \%$ specificity (95\% CI, 57.2\%-98.2\%) in predicting conversion to MS. The PPV and NPV were 97.1\% (95\% CI, 90.1\%-99.2\%) and 30.0\% (95\% CI, 22.7\%-38.5\%), respectively. Using the $40 \%$ threshold of lesions with $\mathrm{CVs}$ and/or 1 lesion with an IR, we achieved $90.4 \%$ sensitivity (95\% CI, 82.6\%-95.5\%) and 35.7\% specificity (95\% CI, 12.8\%-64.9\%), and PPV and NPV were 90.4\% (95\% CI, 86.4\%93.4\%) and $35.7 \%$ (95\% CI, 17.9\%-58.7\%), respectively.

Part 3: DIS + Analysis. The On-line Table shows the sensitivity, specificity, PPV, and NPV, and hazard ratios for DIS + DIT criteria and DIS + DIT alternatives. For DIS $+\mathrm{CVs}$, we selected $2 \mathrm{CVs}$ (rather than 3) to classify patients as having MS because this increased the number of patients who could be diagnosed at baseline, while still ensuring high specificity. Each of the proposed tests resulted in the following number of patients receiving a diagnosis at baseline: DIS + OCB $(n=69)$, DIS + gadolinium $(n=55)$, DIS + IR $(n=48)$, DIS + iron $(n=55)$, DIS +2 CVs $(n=64)$, and DIS + any $(n=83)$.

\section{DISCUSSION}

In our study, we compared the frequency of lesions with CVs and IRs in patients with CIS and MS-mimicking disorders and tested the diagnostic value of these 2 imaging markers using a 3T SWI protocol. We report that clinically acquired SWI can successfully detect CVs and IRs with high interrater agreement.

In our study, the frequency of CVs was notably lower in the non-MS group, and finding at least 1 lesion with an IR achieved $100 \%$ specificity when used to differentiate CIS and non-MS groups. Moreover, finding at least 3 lesions with CVs or 1 lesion 
with an IR on a baseline SWI scan demonstrated high sensitivity and specificity (70\% and $86 \%$, respectively) in predicting conversion to MS in patients with typical CIS followed up for an average of 4 and a half years. Moreover, combining evidence of DIS and the simultaneous presence of iron-positive and iron-negative lesions or the presence of at least 2 lesions with CVs predicted a 2- to 3-fold increased risk of a subsequent MS diagnosis (irrespective of using the radiologic or clinical criteria) and demonstrated increased diagnostic specificity compared with using DIS and positive OCBs, currently used to support the diagnosis of MS in clinical practice.

The International Panel on Diagnosis of MS has identified the study of the CV sign and IRs as a high-priority research area. ${ }^{1}$ The $\mathrm{CV}$ sign has been previously studied using a variety of MR imaging protocols. Some of them, such as FLAIR ${ }^{\star 28}$ require extra postprocessing steps, while others have used a 3D echo-planar sequence with a gadolinium-based contrast agent. ${ }^{13}$ Our protocol used a widely available $3 \mathrm{D}$ gradient-echo sequence without contrast, a strategy that follows the recommendations made by different organizations on the restrictive use of gadolinium-based contrast agents due to convincing evidence indicating the deposition of gadolinium in certain regions of the CNS after repeat administrations. ${ }^{29}$

The use of 3 lesions with CVs for MS diagnosis has previously demonstrated high specificity values of $>90 \%,{ }^{13,30}$ including in the largest multicenter study of the CV sign, which reported high specificity (89\%) when 3 lesions with CVs were used to distinguish MS (including CIS) and non-MS, though the patients with CIS were not followed up longitudinally. ${ }^{23}$ The only previous, prospective study of the CV sign using 3T SWI involved 14 patients and concluded that the CV sign was useful in differentiating MS and non-MS lesions. ${ }^{31}$ In our study of $>100$ patients with CIS, we confirm that an unenhanced SWI sequence, which can be easily implemented in a clinical MR imaging protocol, can offer valuable diagnostic information at the earliest stages of MS.

Similarly, most of the studies that assessed the presence of IRs have been performed using 7T quantitative susceptibility mapping and phase imaging. ${ }^{17,18,32-36}$ Recently, Absinta et $\mathrm{al}^{37}$ compared 7T and $3 \mathrm{~T}$ phase images and found that almost all $7 \mathrm{~T}$ rings were also visible at $3 \mathrm{~T}$. However, quantitative susceptibility mapping and phase imaging are not typically used, or even available, for diagnostic purposes in clinical practice. In our study using SWI sequences, IRs were completely absent in patients who did not have an MS diagnosis, whereas 56\% of patients who fulfilled the 2017 McDonald criteria had at least 1 lesion with an IR.

Moreover, because lesions with IRs are thought to represent the chronic, active stage of lesion evolution, ${ }^{7,37}$ we could speculate that the simultaneous presence of iron-positive and -negative lesions on a single scan provides objective evidence of DIT, similar to the way the simultaneous presence of gadolinium-enhancing and nonenhancing lesions on a single MR imaging scan is used in the $\mathrm{McD}$ onald criteria. Iron dots, on the other hand, were rare, comprising $<10 \%$ of all the lesions. This hypointense signal on SWI might indicate iron aggregates within lesions; ${ }^{38}$ however, it is also possible that in small lesions, IRs appear dotlike due to partial volume effects. Future follow-up of patients included in this study will help us understand the temporal evolution of these lesions in MS.

In our study, when combined with evidence of DIS, the presence of iron-positive and -negative lesions or 2 lesions with CVs had higher specificity compared with OCBs, which had the lowest specificity of all the tests. Although useful diagnostically as an alternative to DIT, OCB testing is invasive and can result in adverse effects, ${ }^{39}$ which cause some patients to refuse to have a lumbar puncture. Specific, noninvasive MR imaging markers have the potential to help diagnose MS without exposing patients to unnecessary risks. Moreover, from the patient perspective, a short (in our study, $<5$ minutes), additional MR imaging sequence would be far more convenient than OCB testing.

One of the strengths of our study is that our patients were scanned before a final diagnosis was reached, reflecting the way the CV sign and IRs would be applied in clinical practice. So far, few studies of the CV sign at 3T have tested its diagnostic value in patients with CIS with follow-up. ${ }^{31,40,41}$ We are not aware of any studies of the diagnostic value of IRs at 3T. Another strength of our study is the easy implementation of our diagnostic criteria in a clinical setting, even by relatively inexperienced raters. Finding a fixed number of lesions with CVs and/or IRs is more practical than using a proportion-based approach, which requires the analysis of all the lesions. Moreover, the low number of lesions required for a diagnosis means this approach can be applied even in patients with a small lesion load.

Our study also had limitations. Our non-MS group was relatively small, and we analyzed only scans from 1 center using a single scanner and protocol. These features mean that our results cannot be generalized to other centers or scanners. We excluded a large proportion of patients from the study, largely due to lack of eligible lesions (supratentorial, $>3 \mathrm{~mm}$ ), which could likely have led to an overestimation of the true frequency of lesions with CVs and IRs in this patient population. Moreover, the use of absolute numbers of lesions with CVs should be further tested in future studies of patients with a full spectrum of MS mimics. This is an important issue, considering that many studies have primarily reported proportion-based diagnostic cut offs and studies comparing the use of absolute lesion numbers versus proportionbased approaches remain inconclusive. ${ }^{13,23}$

It remains to be determined which, if any, gradient-echo sequence would be optimal for the detection of CVs and IRs on the same scan. While the Magnetic Resonance Imaging in Multiple Sclerosis study found that an optimized T2*-weighted protocol led to increased diagnostic sensitivity in a small number of patients compared with SWI, ${ }^{23}$ a further evaluation of clinically available $3 \mathrm{~T}$ imaging protocols is needed. Finally, even with a mean follow-up period of 4 and a half years, some patients did not develop CDMS; only long-term follow-up of these patients will demonstrate the usefulness of SWI in MS diagnosis.

\section{CONCLUSIONS}

Clinically available, 3T susceptibility-weighted MR imaging can successfully visualize CVs and IRs, which appear to be highly specific features of early MS lesions. Our easy-to-implement proposed criteria could be applied in a clinical setting without the need for postprocessing and could be a good alternative to gadolinium for demonstrating DIT or to OCB testing. Future prospective, multicenter studies are needed to confirm our findings of the diagnostic role of the CV sign and IRs in MS. 


\section{ACKNOWLEDGMENTS}

We wish to thank Aran Garcia-Vidal (Vall d'Hebron Research Institute, Barcelona, Spain) for assistance with data organization for this project.

Disclosures: Margareta A. Clarke-RELATED: Grant: European Center For Treatment and Research In Multiple Sclerosis-Magnetic Resonance Imaging in Multiple Sclerosis Fellowship, Comments: 2018 European Center For Treatment and Research in Multiple Sclerosis-Magnetic Resonance Imaging in Multiple Sclerosis Fellow stipend; Consulting Fee or Honorarium: Novartis Pharmaceuticals UK, Comments: speaker honorarium for a talk at a the Novartis-sponsored research meeting. The talk was not related to any research or activities carried out/supported by Novartis; Support for Travel to Meetings for the Study or Other Purposes: European Center for Treatment and Research in Multiple Sclerosis travel grant, paid to the individual, Comments: a young investigators travel grant to attend and present this work at the 35th Congress for the Treatment and Research in Multiple Sclerosis. Deborah Pareto-UNRELATED: Other: teaching honoraria from Novartis and Sanofi-Genzyme. Lucas Pessini-FerreiraUNRELATED: Employment. Hospital Vall d'Hebrón. Georgina Arrambide — UNRELATED: Consultancy: Sanofi Genzyme, Merck, and Roche, Comments: compensation for consulting services or participation in advisory boards*; Grants/Grants Pending: Instituto de Salud Carlos III, Comments: Proyectos de Investigación en Salud, grant P119/01590, Neurofilament light chain levels as a prognostic biomarker of disease evolution and of treatment response in multiple sclerosis*; Payment for Lectures Including Service on Speakers Bureaus: Sanofi Genzyme, Merck, and Novartis, Comments: speaking honoraria*; Travel/Accommodations/Meeting Expenses Unrelated to Activities Listed: Novartis, Roche, Stendhal, and European Center for Treatment and Research in Multiple Sclerosis, Comments: travel expenses for scientific meetings; Other: Novartis, Comments: research support.* Mar Tintoré—UNRELATED: Board Membership: Almirall, Bayer, Biogen-Idec, Genzyme, Merck Serono, Novartis, Roche, Sanofi-Aventis, and Teva Pharmaceuticals; Consultancy: Almirall, Bayer, Biogen Idec, Genzyme, Merck Serono, Novartis, Roche, Sanofi-Aventis, and Teva Pharmaceuticals; Grants/Grants Pending: Biogen Idec, Genzyme, Novartis*; Payment for Lectures Including Service on Speakers Bureaus: Almirall, Bayer, Biogen Idec, Genzyme, Merck Serono, Novartis, Roche, Viela Bio, Sanofi-Aventis, and Teva Pharmaceuticals; Payment for Development of Educational Presentations: Biogen, Merck; Travel/Accommodations/Meeting Expenses Unrelated to Activities Listed: Almirall, Bayer, Biogen Idec, Genzyme, Merck Serono, Novartis, Roche, Sanofi-Aventis, and Teva Pharmaceuticals. Jaume SastreGarriga-RELATED: Grant. European Center for Treatment and Research in Multiple Sclerosis, Comments: European Center for Treatment and Research in Multiple Sclerosis-Magnetic Resonance Imaging in Multiple Sclerosis fellowship*; UNRELATED: Payment for Lectures Including Service on Speakers Bureaus: Genzyme, Biogen, Bayer, Merck, Biopass, Bial Pharmaceuticals, Novartis, Roche, Teva Pharmaceutical Industries, Celgene. Xavier Montalban—UNRELATED: Consultancy: Actelion Pharmaceuticals, Alexion Pharmaceuticals, Bayer, Biogen, Celgene, EMD Serono, Genzyme, Immunic Therapeutics, MedDay Pharma, Merck, Nervgen Pharma, Novartis, Roche, Sanofi Genzyme, Teva Pharmaceutical Industries, TG Therapeutics, EXCEMED, Multiple Sclerosis International Federation and National MS Society. Nikos EvangelouUNRELATED: Consultancy: Biogen, Roche, Comments: member of advisory board; Grants/Grants Pending: Media Research Center, Patient-Centered Outcomes Research Institute, National Institute for Health Research*; Payment for Lectures Including Service on Speakers Bureaus: Biogen, Roche; Payment for Development of Educational Presentations: Biogen, Comments: educational material for use of MRI in MS; Travel/Accommodations/Meeting Expenses Unrelated to Activities Listed: Biogen, Comments: attendance at European Center For Treatment and Research in Multiple Sclerosis. Àlex Rovira—UNRELATED: Board Membership: Novartis, Sanofi-Genzyme, Bayer, Roche, Biogen; Payment for Lectures Including Service on Speakers Bureaus: Bayer, Sanofi-Genzyme, Bracco, Merck Serono, Teva Pharmaceutical Industries, Novartis, Roche, and Biogen; Payment for Development of Educational Presentations: Bayer, Sanofi-Genzyme, Bracco, Merck Serono, Teva Pharmaceutical Industries, Novartis, Roche, and Biogen. *Money paid to the institution.

\section{REFERENCES}

1. Thompson AJ, Banwell BL, Barkhof F, et al. Diagnosis of multiple sclerosis: 2017 revisions of the McDonald criteria. Lancet Neurol 2018;17:162-73 CrossRef Medline

2. Hyun JW, Kim W, Huh SY, et al. Application of the 2017 McDonald diagnostic criteria for multiple sclerosis in Korean patients with clinically isolated syndrome. Mult Scler 2019;25:1488-95 CrossRef Medline

3. Gobbin F, Zanoni M, Marangi A, et al. 2017 McDonald criteria for multiple sclerosis: earlier diagnosis with reduced specificity? Mult Scler Relat Disord 2019;29:23-25 CrossRef Medline
4. Reichenbach JR, Venkatesan R, Schillinger DJ, et al. Small vessels in the human brain: MR venography with deoxyhemoglobin as an intrinsic contrast agent. Radiology 1997;204:272-77 CrossRef Medline

5. Haacke EM, Xu Y, Cheng YC, et al. Susceptibility weighted imaging (SWI). Magn Reson Med 2004;52:612-18 CrossRef Medline

6. Haacke EM, Makki M, Ge Y, et al. Characterizing iron deposition in multiple sclerosis lesions using susceptibility weighted imaging. $J$ Magn Reson Imaging 2009;29:537-44 CrossRef Medline

7. Dal-Bianco A, Grabner G, Kronnerwetter C, et al. Slow expansion of multiple sclerosis iron rim lesions: pathology and $7 \mathrm{~T}$ magnetic resonance imaging. Acta Neuropathol 2017;133:25-42 CrossRef Medline

8. Tallantyre E, Brookes M, Dixon J, et al. Demonstrating the perivascular distribution of MS lesions in vivo with 7-Tesla MRI. Neurology 2008;70:2076-78 CrossRef Medline

9. Tallantyre EC, Morgan PS, Dixon JE, et al. A comparison of 3T and $7 \mathrm{~T}$ in the detection of small parenchymal veins within MS lesions. Investigative Radiol 2009;44:491-94 CrossRef Medline

10. Mistry N, Abdel-Fahim R, Samaraweera A, et al. Imaging central veins in brain lesions with $3-\mathrm{T} T 2^{*}$-weighted magnetic resonance imaging differentiates multiple sclerosis from microangiopathic brain lesions. Mult Scler 2016;22:1289-96 CrossRef Medline

11. Sati P, Oh J, Constable RT, et al; NAIMS Cooperative. The central vein sign and its clinical evaluation for the diagnosis of multiple sclerosis: a consensus statement from the North American Imaging in Multiple Sclerosis Cooperative. Nat Rev Neurol 2016;12:714-22 CrossRef Medline

12. Samaraweera AP, Clarke MA, Whitehead A, et al. The central vein sign in multiple sclerosis lesions is present irrespective of the $\mathrm{T} 2^{*}$ sequence at 3 T. J Neuroimaging 2017;27:114-21 CrossRef Medline

13. Maggi $P$, Absinta $M$, Grammatico $M$, et al. The central vein sign differentiates MS from CNS inflammatory vasculopathies. Ann Neurol 2018;83:283-94 CrossRef Medline

14. Tallantyre EC, Dixon JE, Donaldson I, et al. Ultra-high-field imaging distinguishes MS lesions from asymptomatic white matter lesions. Neurology 2011;76:534-39 CrossRef Medline

15. Campion T, Smith P, Turner B, et al. FLAIR ${ }^{\star}$ for the non-invasive histological diagnosis of multiple sclerosis (S29.003). Neurology 2015;84:(14 Suppl)S29.003

16. Mistry N, Dixon J, Tallantyre E, et al. Central veins in brain lesions visualized with high-field magnetic resonance imaging: a pathologically specific diagnostic biomarker for inflammatory demyelination in the brain. JAMA Neurol 2013;70:623-28 CrossRef Medline

17. Harrison DM, Li X, Liu H, et al. Lesion heterogeneity on high-field susceptibility MRI is associated with multiple sclerosis severity. AJNR Am J Neuroradiol 2016;37:1447-53 CrossRef Medline

18. Chawla S, Kister I, Wuerfel J, et al. Iron and non-iron-related characteristics of multiple sclerosis and neuromyelitis optica lesions at 7T MRI. AJNR Am J Neuroradiol 2016;37:1223-30 CrossRef Medline

19. Wuerfel J, Sinnecker T, Ringelstein EB, et al. Lesion morphology at 7 Tesla MRI differentiates Susac syndrome from multiple sclerosis. Mult Scler 2012;18:1592-99 CrossRef Medline

20. Hosseini Z, Matusinec J, Rudko DA, et al. Morphology-specific discrimination between MS white matter lesions and benign white matter hyperintensities using ultra-high-field MRI. AJNR Am J Neuroradiol 2018;39:1473-79 CrossRef Medline

21. Tintore M, Rovira A, Rio J, et al. Defining high, medium and low impact prognostic factors for developing multiple sclerosis. Brain 2015;138:1863-74 CrossRef Medline

22. Arrambide G, Tintore M, Espejo C, et al. The value of oligoclonal bands in the multiple sclerosis diagnostic criteria. Brain 2018;141:1075-84 CrossRef Medline

23. Sinnecker T, Clarke MA, Meier D, et al; for the MAGNIMS Study Group. Evaluation of the central vein sign as diagnostic imaging biomarker in multiple sclerosis. JAMA Neurol 2019 Aug 19. [Epub ahead of print] CrossRef Medline 
24. Freedman MS, Thompson EJ, Deisenhammer F, et al. Recommended standard of cerebrospinal fluid analysis in the diagnosis of multiple sclerosis: a consensus statement. Arch Neurol 2005;62:865-70 CrossRef Medline

25. Fedorov A, Beichel R, Kalpathy-Cramer J, et al. 3D Slicer as an image computing platform for the Quantitative Imaging Network. Magn Reson Imaging 2012;30:1323-41 CrossRef Medline

26. Hagemeier J, Heininen-Brown M, Poloni GU, et al. Iron deposition in multiple sclerosis lesions measured by susceptibility-weighted imaging filtered phase: a case control study. J Magn Reson Imaging 2012;36:73-83 CrossRef Medline

27. Poser CM, Paty DW, Scheinberg L, et al. New diagnostic criteria for multiple sclerosis: guidelines for research protocols. Ann Neurol 1983;13:227-31 CrossRef Medline

28. Campion T, Smith RJ, Altmann DR, et al. FLAIR* to visualize veins in white matter lesions: a new tool for the diagnosis of multiple sclerosis? Eur Radiol 2017;27:4257-63 CrossRef Medline

29. Guo BJ, Yang ZL, Zhang LJ. Gadolinium deposition in brain: current scientific evidence and future perspectives. Front Mol Neurosci 2018;11:335-35 CrossRef Medline

30. Solomon AJ, Watts R, Ontaneda D, et al. Diagnostic performance of central vein sign for multiple sclerosis with a simplified threelesion algorithm. Mult Scler 2018;24:750-57 CrossRef Medline

31. Kau T, Taschwer M, Deutschmann H, et al. The "central vein sign": is there a place for susceptibility weighted imaging in possible multiple sclerosis? Eur Radiol 2013;23:1956-62 CrossRef Medline

32. Absinta M, Sati P, Gaitan MI, et al. Seven-Tesla phase imaging of acute multiple sclerosis lesions: a new window into the inflammatory process. Ann Neurol 2013;74:669-78 CrossRef Medline
33. Yao B, Bagnato F, Matsuura E, et al. Chronic multiple sclerosis lesions: characterization with high-field-strength MR imaging. Radiology 2012;262:206-15 CrossRef Medline

34. Bagnato F, Hametner S, Yao B, et al. Tracking iron in multiple sclerosis: a combined imaging and histopathological study at 7 Tesla. Brain 2011;134:3602-15 CrossRef Medline

35. Bian W, Harter K, Hammond-Rosenbluth KE, et al. A serial in vivo 7T magnetic resonance phase imaging study of white matter lesions in multiple sclerosis. Mult Scler 2013;19:69-75 CrossRef Medline

36. Hammond KE, Metcalf M, Carvajal L, et al. Quantitative in vivo magnetic resonance imaging of multiple sclerosis at 7 Tesla with sensitivity to iron. Ann Neurol 2008;64:707-13 CrossRef Medline

37. Absinta $\mathrm{M}$, Sati $\mathrm{P}$, Fechner $\mathrm{A}$, et al. Identification of chronic active multiple sclerosis lesions on 3T MRI. AJNR Am J Neuroradiol 2018;39:123338 CrossRef Medline

38. Hametner S, Wimmer I, Haider L, et al. Iron and neurodegeneration in the multiple sclerosis brain. Ann Neurol 2013;74:848-61 CrossRef Medline

39. Engelborghs S, Niemantsverdriet E, Struyfs H, et al. Consensus guidelines for lumbar puncture in patients with neurological diseases. Alzheimers Dement (Amst) 2017;8:111-26 CrossRef Medline

40. Clarke MA, Samaraweera AP, Falah Y, et al. Single Test to ARrive at Multiple Sclerosis (STAR-MS) diagnosis: a prospective pilot study assessing the accuracy of the central vein sign in predicting multiple sclerosis in cases of diagnostic uncertainty. Mult Scler 2020;26:43341 CrossRef

41. Maggi $P$, Absinta $M$, Sati $P$, et al. The “central vein sign” in patients with diagnostic "red flags" for multiple sclerosis: a prospective multicenter 3T study. Mult Scler 2020;26:421-32 CrossRef Medline 